\title{
Assessment and Confirmation of Species Difference in Nonlinear Pharmacokinetics of Atipamezole with Physiologically Based Pharmacokinetic Modeling ${ }^{\llbracket}$
}

\author{
Zheng Li, You Gao, Chunmiao Yang, Yanan Xiang, Wenpeng Zhang, Tianhong Zhang, Ruibin Su, \\ Chuang Lu, and Xiaomei Zhuang
}

State Key Laboratory of Toxicology and Medical Countermeasures, Beijing Institute of Pharmacology and Toxicology, Beijing, China (Z.L., Y.G., C.Y., Y.X., W.Z., T.Z., R.S., X.Z.); and Department of DMPK, Sanofi Company, Waltham, Massachusetts (C.L.)

Received August 29, 2019; accepted October 24, 2019

\section{ABSTRACT}

Atipamezole, an $\alpha_{2}$-adrenoceptor antagonist, displayed nonlinear pharmacokinetics (PK) in rats. The aim of this study was to understand the underlying mechanisms of nonlinear PK in rats and linear PK in humans and develop physiologically based PK models (PBPK) to capture and validate this phenomenon. In vitro and in vivo data were generated to show that metabolism is the main clearance pathway of atipamezole and species differences exist. Where cytochrome P450 (P450) was responsible for the metabolism in rats with a low Michaelis constant, humanspecific UDP-glucuronosyltransferase 2B10- and 1A4-mediated $\mathrm{N}$-glucuronidation was identified as the leading contributor to metabolism in humans with a high $\boldsymbol{V}_{\max }$ capacity. Saturation of metabolism was observed in rats at pharmacologically relevant doses, but not in humans at clinically relevant doses. PBPK models were developed using GastroPlus software to predict the PK profile of atipamezole in rats after intravenous or intramuscular administration of 0.1 to $3 \mathrm{mg} / \mathrm{kg}$ doses. The model predicted the nonlinear PK of atipamezole in rats and predicted observed exposures within 2-fold across dose levels. Under the same model structure, a human PBPK model was developed using human in vitro metabolism data. The PBPK model well described human concentration-time profiles at 10-100 $\mathrm{mg}$ doses showing dose-proportional increases in exposure. This study demonstrated that PBPK is a useful tool to predict human PK when interspecies extrapolation is not applicable. The nonlinear PK in rat and linear PK in human were characterized in vitro and allowed the prospective human PK via intramuscular dosing to be predicted at the preclinical stage.

\section{SIGNIFICANCE STATEMENT}

This study demonstrated that PBPK is a useful tool for predicting human PK when interspecies extrapolation is not applicable due to species unique metabolism. Atipamezole, for example, is metabolized by $\mathrm{P} 450$ in rats and by $\mathrm{N}$-glucuronidation in humans that were hypothesized to be the underlying reasons for a nonlinear $P K$ in rats and linear PK in humans. This was testified by PBPK simulation in this study.

\section{Introduction}

Nonlinear PK profiles are observed in preclinical studies in part because a wide dose range is studied. This sort of nonlinearity makes assessment of the relationships of dose toxicity and dose efficacy difficult due to the occurrence of nondose-proportional exposure increase or decrease. It is therefore very crucial to qualitatively elucidate the underlying mechanisms and quantitatively predict nonlinear PK of a new chemical entity in humans (Fukuchi et al., 2017).

X.Z. was supported by National Science \& Technology Major Special Project on Major New Drug Innovation, China [2018ZX09711003-006 and AWS16J016]. T.Z. was supported by National Science \& Technology Major Special Project on Major New Drug Innovation, China [2018ZX09721003-001-005].

https://doi.org/10.1124/dmd.119.089151.

SThis article has supplemental material available at dmd.aspetjournals.org.
Atipamezole (Fig. 1) is a novel, potent, and selective $\alpha_{2}$-adrenoceptor antagonist devoid of significant interactions with other neurotransmitter receptors. It can rapidly reverse sedation anesthesia induced by $\alpha_{2^{-}}$ adrenoceptor agonists. Hence, in veterinary practice, atipamezole has been commonly used to recover animals from sedation-anesthesia induced by $\alpha_{2}$-adrenoceptor agonists for over two decades (Ewing et al., 1993). Animal studies suggest atipamezole enhanced sexual activity in rats and monkeys (Viitamaa et al., 1995; Pertovaara et al., 2004) and improved behavioral performance of rats subjected to focal cerebral ischemia (Puurunen et al., 2001; Pertovaara et al., 2005). Studies also suggested that atipamezole might have beneficial effects in the recovery from brain damage and might potentiate the anti-Parkinsonian effects of dopaminergic drugs (Pitkänen et al., 2004). Atipamezole is currently being investigated for human application; however, a clear understanding of its disposition is lacking in both animals and humans. Atipamezole has a high clearance and is subject to significant firstpass clearance (Pertovaara et al., 2005); hence, intravascular (i.v.) and

ABBREVIATIONS: AUC, area under the concentration-time curve; CNS, central nervous system; DDI, drug drug interaction; HLM, human liver microsomes; IS, internal standard; IVIVE, in vitro-in vivo extroplation; $K_{m}$, Michaelis constant; $K_{p}$, tissue-to-plasma partition coefficient; LC-MS/MS, liquid chromatography tandem mass spectrometry; MRM, multiple reaction monitor; P450, cytochrome P450; PBPK, physiologically based PK; PK, pharmacokinetic; QC, quality control; $\mathrm{R}_{\mathrm{bp}}$, blood/plasma concentration ratio; RLM, rat liver microsomes; RSD, relative standard deviation; UDPGA, uridine diphosphate glucuronic acid; UGT, UDP-glucuronosyltransferase. 


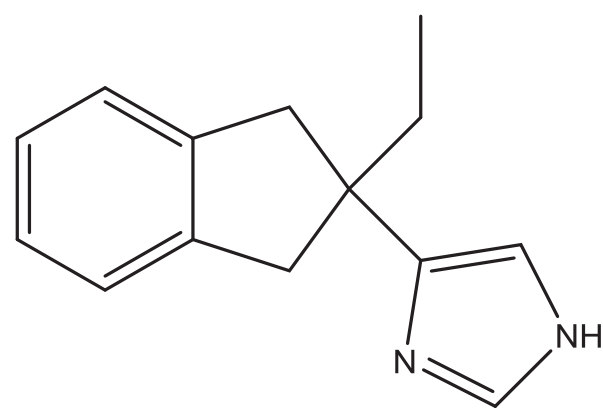

Fig. 1. Chemical structure of atipamezole.

intramuscular (i.m.) routes are considered as potential drug administration routes.

In the literature, human PK of atipamezole was characterized in a clinical study via single ascending i.v. administration at doses of 10 to $100 \mathrm{mg}$ (Karhuvaara et al., 1990). Our preclinical characterization of atipamezole PK showed nonlinear PK in rats, yet linear increases in exposure were reported for humans at a comparable exposure compared with rats [area under the concentration-time curve (AUC) range $\sim 100-4000$ hour $\times \mathrm{ng} / \mathrm{ml}$. When single i.v. administration of atipamezole was given to rats from 0.3 to $3 \mathrm{mg} / \mathrm{kg}$, the systemic plasma clearance of atipamezole decreased with increased dose. Therefore, there was a need to understand the underlying disposition of atipamezole in rats and humans and particularly the nonlinearity to better estimate PK and pharmacodynamics relationship and further build confidence around safety margins and dose escalation scheme for clinical trials with various administration routes (Bachmann and Belloto, 1999; Tachibana et al., 2012; Wu et al., 2014; Chirehwa et al., 2015).

Besides in vitro-in vivo extrapolation, traditional approaches for interspecies PK extrapolation are based on allometric scaling, which is an empirical prediction from animals to obtain human PK parameters (e.g., clearance, volume of distribution) (Tang and Mayersohn, 2006). Those methods can be applied only to drugs with linear PK and no species unique metabolism exits for a given drug. For predicting and understanding saturable PK of a new drug candidate, mechanistic modeling and simulation approaches across species are increasingly being applied (Huang and Rowland, 2012; Shebley et al., 2018). Among the modeling and simulation approaches, physiologically based PK (PBPK) model is a powerful tool to quantitatively delineate how certain extrinsic and intrinsic factors might influence the nonproportional systemic exposures. PBPK modeling is based on drug-dependent physicochemical and PK parameters as well as drug-independent physiologic systemic parameters to capture the kinetics and dynamics of drug absorption, distribution, metabolism, and excretion (Rowland et al., 2011; Liu et al., 2014; Jones et al., 2015; Zhuang and Lu, 2016; Li et al., 2018b). With broad application of PBPK modeling and many successful examples of predicting human PK and Drug Drug Interaction (DDI), it is particularly useful in capturing linear and nonlinear processes simultaneously (Dong et al., 2011; Chen et al., 2013).

The purpose of this study is to use a PBPK modeling approach to predict both rat and human PK and predict exposures in humans after various routes of administration. To achieve this, key in vitro and in vivo data for atipamezole were generated in rats (in vitro and in vivo) and in humans (in vitro only) to understand the respective metabolism mechanisms and tissue partitioning. Then a bottom-up combined with top-down PBPK model was developed and applied in the assessment of nonlinear $\mathrm{PK}$ in rats and identifying the mechanism that led to the nonlinearity. Ultimately, a PBPK model was built to predict human PK using all available information from preclinical in vitro and in vivo studies, as well as the learnings from the rat PBPK model.

\section{Materials and Methods}

Chemicals and Reagents. Atipamezole hydrochloride was supplied by chemical synthesis laboratory of our Institute (Beijing, China) with purity greater than 99\% (Supplemental Fig. 1 is the NMR spectrum) (Li et al., 2019). Midazolam, 1'-OH-midazolam, phenacetin, acetaminophen, diclofenac, $\mathrm{S}$-mephenytoin, 4-OH-diclofenac, 4-OH-mephenytoin, bupropion, $\mathrm{OH}$ bupropion, amodiaquine, $\mathrm{N}$-desethylamodiaquine, dextromethorphan, and dextrorphan were all purchased from Sigma-Aldrich (St. Louis, MO). Human liver microsomes (150-donor pool, mixed sex), male rat liver microsomes, and recombinant UDP-glucuronosyltransferase (UGT) Supersomes (UGT1A1, 1A3, 1A4, 1A6, 1A8, 1A9, 2B4, 2B7, 2B10, 2B15, and 2B17) were purchased from Corning Life Sciences (Tewksbury, MA). NADPH was purchased from Roche Life Science (Basel-Stadt, Switzerland). Other reagents were of high-pressure liquid chromatography grade or better.

NADPH- and Uridine Diphosphate Glucuronic Acid-Dependent Hepatic Clearance in Liver Microsomes. The P450-mediated metabolic stability was conducted in incubations containing $1 \mu \mathrm{M}$ atipamezole in rat or pooled human liver microsomes $(0.5 \mathrm{mg} / \mathrm{ml})$ in $100 \mathrm{mM}$ potassium phosphate buffer with $5 \mathrm{mM}$ $\mathrm{MgCl}_{2}, \mathrm{pH}$ 7.4. The mixture was preincubated at $37^{\circ} \mathrm{C}$ for 5 minutes. NADPH (final concentration of $1 \mathrm{mM}$ ) was added to initiate the reactions. For UGTmediated metabolic clearance assays, alamethicin at final concentration of $25 \mu \mathrm{g} / \mathrm{mg}$ protein, $1 \mu \mathrm{M}$ atipamezole, and rat or human liver microsomes in $100 \mathrm{mM}$ potassium phosphate buffer ( $\mathrm{pH}$ 7.4) were mixed on ice for 15 minutes. The mixture was then preincubated at $37^{\circ} \mathrm{C}$ for 5 minutes. Uridine diphosphate glucuronic acid (UDPGA; final concentration of $2.5 \mathrm{mM}$ ) was added to initiate the reactions. For combined NADPH- and UDPGA-mediated metabolism assays, the pooled liver microsomes of rat or human and drug solutions were treated, as described above, for the glucuronidation assay. The reactions were initiated by adding mixture of NADPH $(1 \mathrm{mM})$ and UDPGA $(2.5 \mathrm{mM})$, final concentrations. Aliquots of the incubation were removed at $0,5,15$, and 30 minutes and diluted in $4 \times$ volume of chilled acetonitrile containing internal standard (IS) to stop the reactions. After centrifugation at $13,000 \mathrm{~g}$ for 10 minutes, the supernatant was collected and stored at $-20^{\circ} \mathrm{C}$ until liquid chromatography tandem mass spectrometry (LC-MS/MS) analysis. The in vitro $t_{1 / 2}$ in liver microsomal incubation was calculated from the semilog plot of atipamezole concentration remaining versus incubation time, and intrinsic clearance $\left(\mathrm{CL}_{\text {int }}, \mathrm{ml} / \mathrm{min}\right.$ per kilogram) was calculated as follows:

$$
C L_{\text {int }}=\frac{0.693}{\text { in vitro } t_{1 / 2}} \times \frac{m l \text { incubation }}{m g \text { microsomes }} \times \frac{45 \mathrm{mg} \text { microsomes }}{g \text { liver }} \times \frac{X g \text { liver }}{\mathrm{kg} \mathrm{BW}}
$$

For rats and humans, $\mathrm{X}$ values of 40 and 25.7 were used, respectively (Davies and Morris, 1993).

The conversion of $\mathrm{CL}_{\text {int }}$ to hepatic clearance $\mathrm{CL}_{\mathrm{h}}$ by a well-stirred model is described previously (Houston, 1994):

$$
C L_{h}=\frac{Q_{h} \times C L_{i n t}}{Q_{h}+C L_{i n t}}
$$

Identification of UGT Involvement in Metabolism of Atipamezole. To identify the hepatic UGTs involved and understand the difference in metabolizing enzymes responsible for the metabolism of atipamezole in humans, recombinant UGT of UGT1A1, 1A3, 1A4, 1A6, 1A8, 1A9, 2B4, 2B7, 2B10, 2B15, and 2B17 were incubated $(0.5 \mathrm{mg} / \mathrm{ml}$ protein with $1 \mu \mathrm{M}$ atipamezole $)$ in a similar way as human liver microsomes (HLM) described above. The reaction product after incubation and protein precipitation was analyzed on LC-MS/MS for the formation of glucuronides.

Measurement of Michaelis Constant in Rat Liver Microsomes Using Substrate Depletion Method. Due to the lack of authentic standards of atipamezole metabolites, the Michaelis constant $\left(K_{m}\right)$ of atipamezole toward P450-mediated biotransformation in rat liver microsomes (RLM) was obtained using a substrate depletion approach (Obach and Reed-hagen, 2002; Nath and Atkins, 2006). Various concentrations of atipamezole $(0.32,0.63,1.25,2.5,5,10$, $20 \mu \mathrm{M})$ and rat liver microsomes $(0.5 \mathrm{mg} / \mathrm{ml})$ in $100 \mathrm{mM}$ potassium phosphate buffer ( $\mathrm{pH}$ 7.4) with $5 \mathrm{mM} \mathrm{MgCl}{ }_{2}$ were preincubated at $37^{\circ} \mathrm{C}$ for 5 minutes. NADPH ( $1 \mathrm{mM}$, final concentration) was added to initiate the reactions. Aliquots were removed from incubations with various initial atipamezole concentrations at $0-, 2-, 5-, 10-, 15-$, and 30-minute time points. The reactions were terminated by 

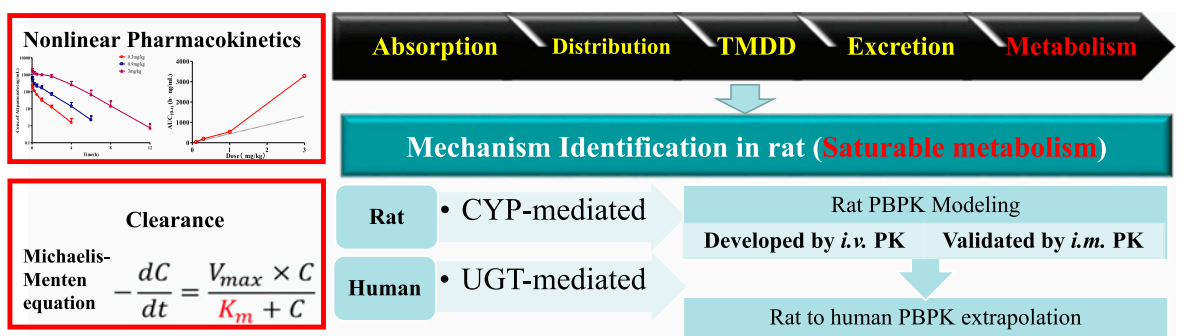

Mechanism Identification in rat $(\mathrm{S}$

m)

Rat $\cdot$ CYP-mediated
Human - UGT-mediated
$\begin{gathered}\text { Perfusion } \\ \text { Limited }\end{gathered}$
$\begin{gathered}\text { Use } K_{p, u u} \\ \text { extrapolated to } \\ \text { human }\end{gathered}$

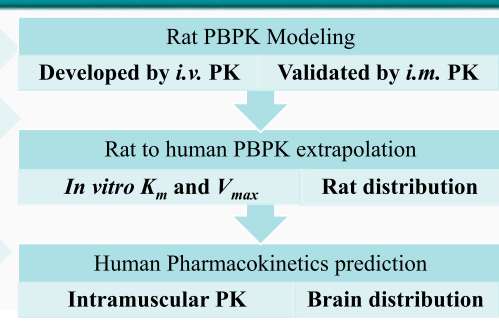

Intramuscular PK
Fig. 2. Work flow scheme of the atipamezole PBPK model construction, verification, and prediction.

Distribution
$\frac{K_{p, \text { rat }}}{f_{\text {pu,human }}}=\frac{K_{p, \text { human }}}{f_{\text {up,rat }}}$

diluting the aliquots in $4 \times$ volume of chilled acetonitrile containing IS. The samples were processed for analysis by mass spectrometry, as stated below. The log-transformed percentage remaining of atipamezole was plotted against each time point for each incubation group to obtain the substrate depletion rate constants $\left(k_{\mathrm{dep}}\right) . K_{m}$ value was then determined from the inflection point of a plot of the $k_{\text {dep }}$ values versus the initial atipamezole concentrations in a semilog plot following eq. 3 :

$$
k_{d e p}=k_{d e p([s]=0)} \times\left(1-\frac{[s]}{[s]+K_{m}}\right)
$$

where $[S]$ is the substrate concentration, $k_{\mathrm{dep}([\mathrm{S}]=0)}$ represents the theoretical maximum substrate depletion rate constant at an infinitely low-substrate concentration, and $K_{m}$ is the $K_{m}$

Enzyme Kinetic Parameters for UGT-Mediated Metabolism in HLM. A metabolite profiling study indicated that atipamezole is mainly metabolized by UGT in HLM and generates a single N-glucuronidation metabolite. The enzyme kinetic parameters $\left(K_{m}\right.$ and $\left.V_{\max }\right)$ of atipamezole in HLM were obtained by determining the formation of the UGT-conjugation metabolite at different atipamezole concentrations. Similar procedure as described above for the HLM clearance assay was applied in a 5-minute incubation with initial concentration of $0.32,0.63,1.25,2.5,5,10$, and $20 \mu \mathrm{M}$. The amount of UGT-conjugated metabolite formed (picomoles per minute per milligram protein) was calculated in the reference of known amount of atipamezole disappearance, as described by eq. 4:

$$
\frac{d_{M}}{d_{t}}=\frac{v_{\max } \times[s]}{K_{m}+[s]}
$$

The data were analyzed with the nonlinear analysis to generate $K_{m}$ and $V_{\max }$ values (Pharsight, Sunnyvale, CA).

Nonspecific Protein Binding. The extent of protein binding of atipamezole to plasma and liver microsomes of rat and human, and major tissue homogenates of rat (liver, kidney, lung, brain, fat, spleen, heart, gonad, and muscle) were determined using a 48-well Rapid Equilibrium Dialysis device (Thermo Bioscience, Woburn, MA). All rat tissues were homogenized in $4 \times$ volume (w/v) of PBS (pH 7.4). Rat or human plasma was spiked with atipamezole at three concentrations $(1,3$, and $10 \mu \mathrm{M})$. Rat or human liver microsomes $(0.5 \mathrm{mg} / \mathrm{ml}$ protein) and other tissue homogenates were spiked with atipamezole at final concentration of $1 \mu \mathrm{M}$. Aliquots of $200 \mu \mathrm{l}$ spiked biologic matrices were loaded into the donor side of a dialysis apparatus, and $350 \mu \mathrm{l}$ buffer was added to the receiving side. The apparatus was incubated in a shaking incubator at $37^{\circ} \mathrm{C}$ for 4 hours. After incubation, the samples from donor and receiving chambers were diluted in the same matrixes in the other chamber, so the final matrix composition was the same. The resulting samples were precipitated in acetonitrile with IS. The atipamezole concentrations were determined by LC-MS/MS

The unbound fraction of atipamezole in plasma or liver microsomes $\left(f_{u, x}\right)$ was calculated as shown in eq. 5 , and unbound fraction in the tissue homogenate $\left(f_{u, t}\right)$ was calculated according to eq. 6 .

$$
\begin{gathered}
f_{u, x}=\text { Conc }_{\text {buffer chamber }} / \text { Conc }_{\text {plasma/liver microsomes chamber }} \\
f_{u, t}=\text { Conc }_{\text {buffer chamber }} / \text { Conc }_{\text {tissuehomogenate chamber }}
\end{gathered}
$$

The measured unbound fractions would be higher when a tissue is homogenized and diluted in buffer; the unbound fractions in undiluted tissues $\left(f_{u, t i s s u e}\right)$ were calculated using eq. 7 .

$$
\text { Undiluted } f_{u, \text { tissue }}=\frac{1 / D}{\left(\left(1 / f_{u, \text { tissue homogenate }}\right)-1\right)+1 / D}
$$

where $D$ represents the fold of dilution factor in tissue homogenates. The free fraction of plasma and undiluted tissue are used in tissue-to-plasma exposure ratio calculation

Blood to Plasma Partitioning. The blood/plasma concentration ratio $\left(\mathrm{R}_{\mathrm{bp}}\right)$ of atipamezole was determined by incubating the compound with fresh whole blood from rats and humans. Atipamezole (final concentration $1 \mu \mathrm{M}$ ) was added to whole blood and an equal volume of plasma isolated from the same blood that served as a control for the whole blood concentration. The spiked whole blood and plasma were incubated at $37^{\circ} \mathrm{C}$ for 1 hour. After incubation, the blood was centrifuged at $2000 \mathrm{~g}$ for 10 minutes, and $50 \mu \mathrm{l}$ aliquots of plasma were removed. All incubations were performed in triplicate. The concentrations of atipamezole in plasma separated from the blood and plasma spiked directly with atipamezole were determined by LC-MS/MS. $\mathrm{R}_{\mathrm{bp}}$ was calculated by dividing the concentration in the plasma spiked directly with atipamezole by the concentration in the plasma separated from blood after incubation.

Disposition and Tissue Distribution of Atipamezole in Rats. Male SD rats $(200-220 \mathrm{~g})$ were obtained from Beijing Vital River Laboratory Animal Technology (Beijing, China). Animals were housed in a temperature- and humidity-controlled room with a 12-hour light/dark cycle. They were fasted 12 hours prior to the experiments and had ad libitum access to water. The animal experiments were conducted in the Beijing Center for Drug Safety Evaluation and according to a protocol approved by the Institutional Animal Care and Use Committee of the Center, which followed the guidelines of the Association for Assessment and Accreditation of Laboratory Animal Care International. The animal protocol number is IACUC-2017-053. For PK investigation, 36 rats were randomly divided into six groups (each group consisting of six rats). Three groups were given i.v. of atipamezole solution via the tail vein at the doses of $0.3,0.9$, and $3 \mathrm{mg} / \mathrm{kg}$. The other three groups were given i.m. of atipamezole solution at the doses of $0.1,1$, and $3 \mathrm{mg} / \mathrm{kg}$. Atipamezole was formulated in saline. For all groups, at sampling times of $0,0.033,0.083,0.25,0.5,1,2,4,6,8,10,12$, and 14 hours after dosing, $0.2 \mathrm{ml}$ blood samples were collected into heparinized polypropylene tubes. The plasma samples were collected after centrifugation of the blood samples at $2000 \mathrm{~g}$ for 10 minutes and stored at $-20^{\circ} \mathrm{C}$ until analysis using an established LC-MS/MS method.

For tissue distribution study, 24 male rats (six animals for each time point) were dosed (i.m.) with the atipamezole solution at $1 \mathrm{mg} / \mathrm{kg}$. The animals were anesthetized and exsanguinated at $0.25,1,4$, and 24 hours postdose. After perfusion with PBS via ventriculus sinister for 2 minutes, major tissues (liver, kidney, lung, brain, adipose, spleen, heart, gonad, and muscle) were harvested, rinsed with cold saline, and blotted dry with filter paper. Tissue samples were homogenized and atipamezole quantitated along with plasma samples using the LC-MS/MS method. The tissue-to-plasma concentration ratios were calculated by dividing tissue concentration over plasma concentration at the indicated time $(0.25,1,4$, and 24 hours postdose $)$ and referred as the tissue-to-plasma partition coefficient $\left(K_{p}\right)$. 
TABLE 1

Physicochemical and Absorption, Distribution, Metabolism, Excretion parameters of atipamezole used for PBPK models

\begin{tabular}{|c|c|c|}
\hline Parameter & Value & Source \\
\hline Molecular weight (g/mol) & 212.3 & GastroPlusTM 9.6 \\
\hline $\mathrm{p} K \mathrm{a}$ & $7.08,13.33$ & GastroPlusTM 9.6 \\
\hline $\operatorname{LogD}$ at $\mathrm{pH} 7.4$ & 3.1 & GastroPlusTM 9.6 \\
\hline Solubility at pH $8.98(\mathrm{mg} / \mathrm{ml})$ & 0.17 & GastroPlusTM 9.6 \\
\hline $\mathrm{R}_{\mathrm{bp}}$ in rat & 1.07 & Measured in this study \\
\hline $\mathrm{R}_{\mathrm{bp}}$ in human & 0.77 & Measured in this study \\
\hline$f_{\mathrm{u}, \mathrm{p}}$ and $f_{\mathrm{u}, \text { mic }}$ of rat & $0.085,0.38$ & Measured in this study \\
\hline$f_{\mathrm{u}, \mathrm{p}}$ and $f_{\mathrm{u}, \mathrm{mic}}$ of human & $0.067,0.59$ & Measured in this study \\
\hline$K_{\mathrm{m}}$ of rat, human metabolizing enzyme $(\mu \mathrm{M})$ & $1.70(361 \mathrm{ng} / \mathrm{ml}), 2.89(614 \mathrm{ng} / \mathrm{ml})$ & Measured in this study \\
\hline$V_{\max }$ of rat, human metabolizing enzyme $[\mathrm{nmol} / \mathrm{min}$ per milligram (protein)] & $0.131,1.07$ & Measured in this study \\
\hline Population information & Caucasian, males, 23-yr-old, height 182 , weight $73 \mathrm{~kg}$ & Karhuvaara et al., 1990 \\
\hline
\end{tabular}

Brain Distribution at Different Dosage. Thirty-six male rats were divided into three groups and dosed with the atipamezole solution at $0.3,1$, and $3 \mathrm{mg} / \mathrm{kg}$ i.m. Animals were anesthetized and then exsanguinated at $0.25,1,4$, and 24 hours after dosing (three animals for each time point). Plasma and brain were harvested and processed with the same methods described above for atipamezole concentration determination.

Sample Process and Analysis Methods. Plasma, blood, microsomal, and tissue homogenate samples were precipitated with $4 \times$ volume of acetonitrile, followed by centrifugation to remove protein. The acetonitrile contains propranolol (30 ng/ml) as IS. The concentrations of atipamezole in all samples were determined by a reported LC-MS/MS method (Li et al., 2019) using a multiple reation moitor (MRM)positive mode with mass transition $(\mathrm{m} / \mathrm{z}$ ) of 213.1 to117.0. The method fulfilled with pre-set acceptance criteria (in-house analytical method validation protocol), including quality control (QC) precision (relative standard deviation (RSD) less than 15\%), QC accuracy (85\%-115\%), and stability (precision and bias less than $\pm 15 \%$ for three cycles of freeze/thaw). Long-term stability was tested at -20 and $-40^{\circ} \mathrm{C}$ for 4 weeks, and short-term stability was tested for 24 hours at room temperature. The calibration/standard curve linearity ranges 0.5 to $1000 \mathrm{ng} / \mathrm{ml}$ with correlation coefficient $r^{2}>0.99$. The method met the acceptance criteria according to the bioanalytical method validation standard established by the Food and Drug Administration. The PK parameters for atipamezole were calculated by noncompartmental analysis using WinNonlin Phoenix v6.3 (Pharsight).

Atipamezole GastroPlus PBPK Model. A full PBPK model was constructed for atipamezole mainly using bottom-up and top-down approach (Jamei et al., 2009) in GastroPlus simulator (version 9.6). The work flow of the model construction, verification, and prediction is presented in Fig. 2. PBPK model was initially developed for a simple $\mathrm{PK}$ profile of atipamezole in rats via i.v. route at low dose $(0.3 \mathrm{mg} / \mathrm{kg})$, which only includes clearance and distribution processes. Key parameters (i.e., physicochemical parameters, $\mathrm{R}_{\mathrm{bp}}, \mathrm{f}_{\mathrm{u}, \mathrm{p}}, \mathrm{f}_{\mathrm{u}, \mathrm{mic}}$ ), $K_{m}$ value generated from RLM incubation, $V_{\max }$ value back-calculated from in vivo clearance, and measured $K_{p}$ values of individual tissues were as input information are presented in Tables 1 and 2 . Then the nonlinear PK profiles of atipamezole in rats via i.v. administrative across ascending dosages were verified with observed data from rat PK studies. In the next step, the PBPK model was employed for rat i.m. PK by alternating the dosing route. After nonlinear PK behaviors of rats were successfully observed with both i.v. and i.m. dosing routes, this PBPK model was extrapolated to humans using human-specific parameters, that is, in vitro enzyme kinetic data combined with tissue distribution profiles converted from $K_{p}$ values obtained in rats corrected with plasma-binding data (Table 2). Populationdependent physiologic parameters in the Population Estimates for Age-Related Physiology module of GastroPlus v9.7 were used in our human PBPK model. Customized information, such as age, gender, height, and body weight in a Caucasian population, was set up to match the patient characterization in a published clinical trial that was used to verify our human model (Karhuvaara et al., 1990).

Statistical Analysis. In in vitro study, replicated incubations/samples were performed $(n=3)$, and all positive and negative control groups were included simultaneously. In in vivo study, replicated animals/samples were performed $(n=$ $6)$. All measured data were presented as means \pm S.D. For the comparison of measured $K_{p}$ values of individual tissues and their predicted $K_{p}$ values using the GastroPlus software (Table 2), ratio of two values beyond $0.5-2$ is defined as significant difference. For accuracy assessment of a model simulation, acceptance is set to be within 2-fold. For comparison between groups, the statistically significant difference is set for a $P$ value of less than 0.05 using analysis of variance, with the Student's $t$ test.

\section{Results}

In Vitro Metabolic Stability of Atipamezole. Atipamezole was found to be metabolized in RLM and HLM to different extents in presence of cofactors of P450s (NADPH) and/or UGTs (UDPGA). The percentages of atipamezole remaining at various time points are presented in Fig. 3. The turnover of atipamezole in RLM only occurred in the presence of NADPH; no decrease of parent drug concentration was observed in the presence of UDPGA. Coincubation with both cofactors had similar depletion rate as the NADPH only (Fig. 3A). In

TABLE 2

Tissue partitions $\left(K_{\mathrm{p}}\right)$ of atipamezole measured in rats and the extrapolated values in human PBPK modeling

\begin{tabular}{|c|c|c|c|c|c|}
\hline Tissue & Measured $K_{p}$ of Rat $(n=6)$ & $f_{\mathrm{ut}}(\%)(n=3)$ & $K_{\text {puu }}$ of Rat $(n=6)$ & Transformed $K_{p}$ of Human ${ }^{a}$ & Predicted $K_{p}$ of $\mathrm{Rat}^{b}$ \\
\hline Brain & $4.4 \pm 0.6$ & $3.7 \pm 0.2$ & $1.9 \pm 0.5$ & 3.5 & $13.77 *$ \\
\hline Adipose & $0.4 \pm 0.2$ & $2.9 \pm 0.2$ & $0.1 \pm 0.06$ & 0.3 & $7.79 *$ \\
\hline Spleen & $2.9 \pm 0.6$ & $3.7 \pm 0.03$ & $1.4 \pm 0.5$ & 2.3 & 4.12 \\
\hline Heart & $1.3 \pm 0.6$ & $2.4 \pm 0.1$ & $0.4 \pm 0.03$ & 1.0 & $3.77 *$ \\
\hline Lung & $6.1 \pm 2.3$ & $1.8 \pm 0.08$ & $1.3 \pm 0.4$ & 4.8 & 6.07 \\
\hline Muscle & $0.6 \pm 0.3$ & $2.9 \pm 0.5$ & $0.2 \pm 0.08$ & 0.5 & $2.65^{*}$ \\
\hline Kidney & $11.4 \pm 4.4$ & $1.0 \pm 0.05$ & $1.4 \pm 0.5$ & 9.0 & 6.62 \\
\hline Liver & $23 \pm 8.9$ & $0.02 \pm 0.01$ & $0.06 \pm 0.02$ & 18.1 & $6.34 *$ \\
\hline Reproductive organ & $4.7 \pm 4$ & $2.7 \pm 0.1$ & $1.5 \pm 0.6$ & 3.7 & 6.63 \\
\hline
\end{tabular}

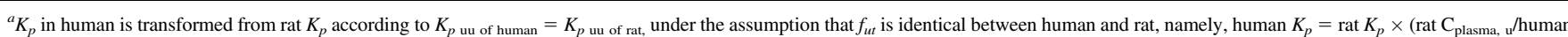
$\mathrm{C}_{\text {plasma, u). }}$.

${ }^{b}$ Predicted $K_{p}$ in rat is obtained using Lukacova tissue-plasma partition method.

*Represents the ratio of predicted $K_{p}$ of measured $K_{p}$ is beyond 2-fold deviation. 
A

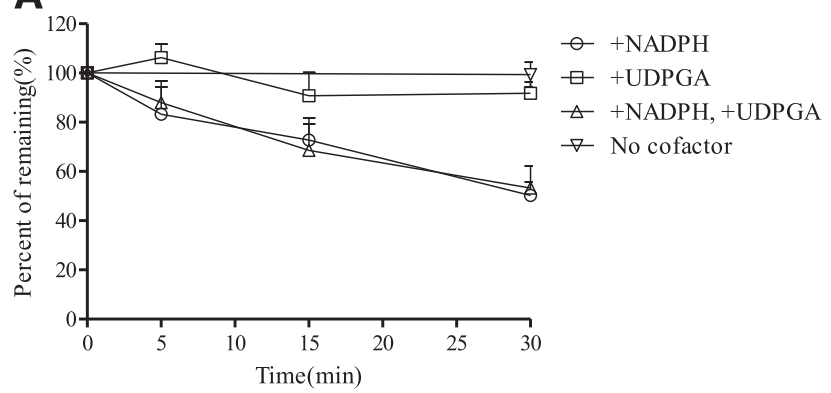

B

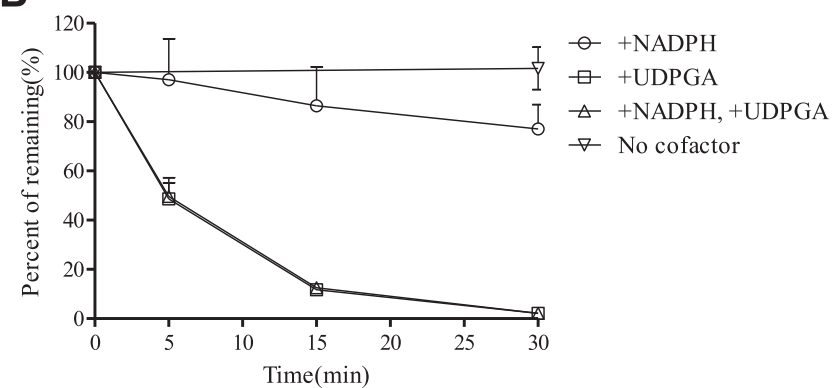

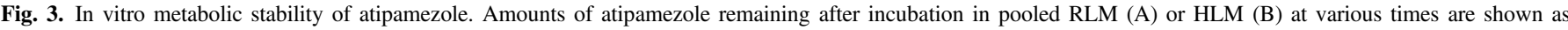
a percentage compared with the amount at time zero. The values represent as means \pm S.D. $(n=3)$.

HLM, the disappearance rate of atipamezole is much greater in the presence of UDPGA than in the presence of NADPH. Coincubation with both cofactors led to almost no increase in turnover rate, as UDPGA alone suggested the direct glucuronidation was the major clearance pathway in HLM (Fig. 3B). The intrinsic clearance and extrapolated hepatic clearance of atipamezole were calculated from data presented in Fig. 3.

In RLM, the hepatic clearance of atipamezole driven by P450 was $31.7 \pm 0.8 \mathrm{ml} / \mathrm{min}$ per kilogram, which was close to rat in vivo clearance $(39.9 \pm 5.8 \mathrm{ml} / \mathrm{min}$ per kilogram). Additionally, an earlier metabolism profiling study in rats detected little parent drug in urine and bile, and circulating oxidized metabolites were mainly presented in bile (data not presented). That confirmed hepatic metabolism is the major clearance pathway for atipamezole in rats catalyzed predominantly by $\mathrm{P} 450$.

In HLM, UGT-mediated hepatic clearance $(19.4 \pm 0.02 \mathrm{ml} / \mathrm{min}$ per kilogram) played a more important role compared with that by $\mathrm{P} 450$ (9.7 $\pm 1.8 \mathrm{ml} / \mathrm{min}$ per kilogram). With the presence of both NADPH and UDPGA, the hepatic clearance $(19.3 \pm 0.04 \mathrm{ml} / \mathrm{min}$ per kilogram) is almost identical to that of only UDPGA presence as a cofactor (Table 3 ). UGT-mediated hepatic clearance is also close to the in vivo clearance of atipamezole reported in humans $(21.3 \pm 2.8 \mathrm{ml} / \mathrm{min}$ per kilogram $)$ (Karhuvaara et al., 1990), as well as to human hepatic liver blood flow (20.7 ml/min per kilogram) (Davies and Morris, 1993). These results demonstrated that hepatic metabolism is the leading elimination route of atipamezole in humans catalyzed primarily by UGT. The in vitro and in vivo metabolite identification has also been conducted in HLM and RLM with NADPH or UDPGA as cofactors and bile from BDC rat to testify the liver metabolism (Supplemental Figs. 2-6, Li et al., 2018a). Three oxidation metabolites were identified in RLM and HLM in the presence of NADPH. In the bile of BDC rat, the same three oxidation metabolites were excreted. Additionally, only a single UGT-conjugated metabolite was found in HLM with UDPGA.

Recombinant UGTs Involved in Human Hepatic Metabolism. Among 11 rUGTs tested, only UGT2B10 and 1A4 were identified to contribute to the hepatic metabolism of atipamezole. Based on equal molar incubations, rUGT2B10 played a more important role than rUGT1A4 at an approximately 15:1 ratio.

Michaelis Constant of Atipamezole in RLM and HLM. Two methods were used to estimate the $K_{m}$ of atipamezole in RLM and HLM due to the different metabolizing enzyme involved in the biotransformation of atipamezole in in rats and humans and lack of metabolite standards. In RLM, the $k_{\mathrm{dep}}$ values at various initial concentrations were determined using a substrate depletion assay (Obach and Reed-Hagen, 2002; Nath and Atkins 2006). The plot of in vitro depletion rate constants versus substrate concentration for atipamezole in RLM is shown in Fig. 4A. The obtained total $K_{m}$ value in RLM was $1.7 \pm$ $0.03 \mu \mathrm{M}(361 \pm 6 \mathrm{ng} / \mathrm{ml})$. The enzyme kinetic parameters of atipamezole in HLM were determined using the N-glucuronidation metabolite formation rate. The $K_{m}$ and $V_{\max }$ values obtained from the Michaelis-Menten plot were $2.89 \pm 0.27 \mu \mathrm{M}(614 \pm 57 \mathrm{ng} / \mathrm{ml})$ and $1.07 \pm 0.03 \mathrm{nmol} / \mathrm{min}$ per milligram protein, respectively (Fig. 4B).

Unbound Fractions of Atipamezole in Plasma. Atipamezole was tested in rat and human plasma at $37^{\circ} \mathrm{C}$ for 4 hours and found to be stable (with recoveries of $96.7 \%-113.8 \%$ ). Unbound fractions of atipamezole in rat or human plasma were determined to identify the potential of protein-binding effect on nonlinear PK. Our results showed no obvious species difference in the unbound fraction in plasma; the atipamezolefree fraction was 0.085 for rats and 0.067 for humans. Moreover, the unbound fractions in rat or human plasma were concentration independent (Table 4).

Nonlinear PK Behavior of Atipamezole in Rats. Mean atipamezole plasma concentration-time profiles in rats after various i.v. (Fig. 5A) and i.m. (Fig. 5B) doses are presented in Fig. 5. Existence of marked nonlinear $\mathrm{PK}$ at $3 \mathrm{mg} / \mathrm{kg}$ is manifested by the dose-normalized AUC profiles in both i.v. (Fig. 5C) and i.m. (Fig. 5D). PK parameters (Table 5) showed $\sim 3$-fold increase in dose $(\sim 1-3 \mathrm{mg} / \mathrm{kg})$ resulted in 7.7- and 6.1-fold increase in AUC after i.v. and i.m., respectively, indicating a superproportional dose-exposure relationship. Total plasma clearance after i.v. dosing decreased notably when dose increased from 0.3 to $3 \mathrm{mg} / \mathrm{kg}$ (i.e., from 39.96 to $13.96 \mathrm{ml} / \mathrm{min}$ per kilogram).

TABLE 3

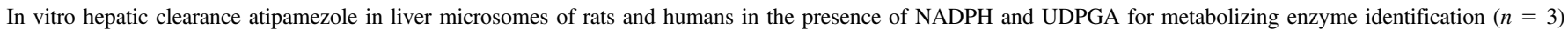

The values represent as means \pm S.D. $(\mathrm{n}=3)$.

\begin{tabular}{|c|c|c|c|c|c|c|}
\hline \multirow{2}{*}{ Metabolizing Enzyme } & \multicolumn{3}{|c|}{ HLM } & \multicolumn{3}{|c|}{ RLM } \\
\hline & $t_{1 / 2}(\min )$ & $\mathrm{Cl}_{\text {int }}(\mathrm{ml} / \mathrm{min}$ per kilogram $)$ & $\mathrm{Cl}_{\mathrm{h}}(\mathrm{ml} / \mathrm{min}$ per kilogram$)$ & $t_{1 / 2}(\min )$ & $\mathrm{Cl}_{\text {int }}(\mathrm{ml} / \mathrm{min}$ per kilogram $)$ & $\mathrm{Cl}_{\mathrm{h}}(\mathrm{ml} / \mathrm{min}$ per kilogram $)$ \\
\hline NADPH & $93.5 \pm 33.8^{* *}$ & $19.5 \pm 6.7^{* *}$ & $9.7 \pm 1.8^{* *}$ & $34.1 \pm 10.1$ & $79.8 \pm 22.6$ & $31.9 \pm 3.9$ \\
\hline UDPGA & $5.4 \pm 0.1$ & $295.9 \pm 5.4$ & $19.4 \pm 0.02$ & 1 & 1 & 1 \\
\hline NADPH + UDPGA & $5.4 \pm 0.2$ & $295.2 \pm 9.9$ & $19.3 \pm 0.04$ & $33.4 \pm 2.0$ & $75.0 \pm 4.8$ & $31.7 \pm 0.8$ \\
\hline
\end{tabular}

$* * * P<0.01$ compared with NADPH + UDPGA group. 
A

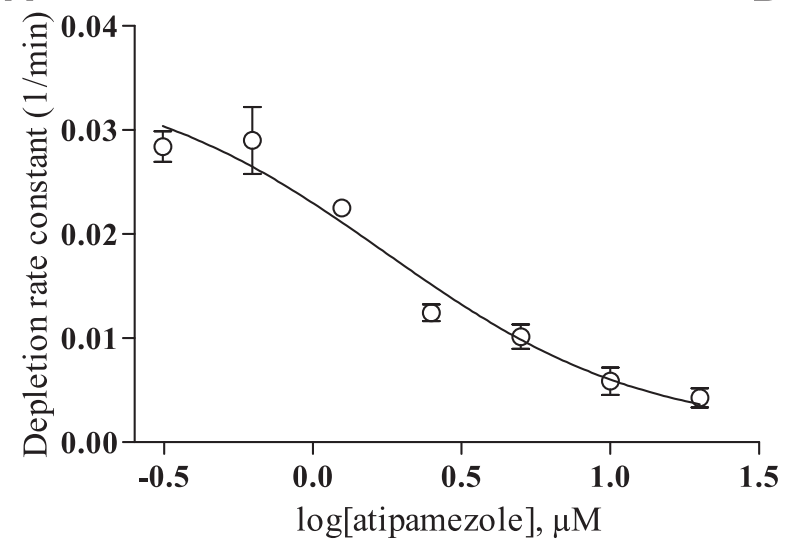

B

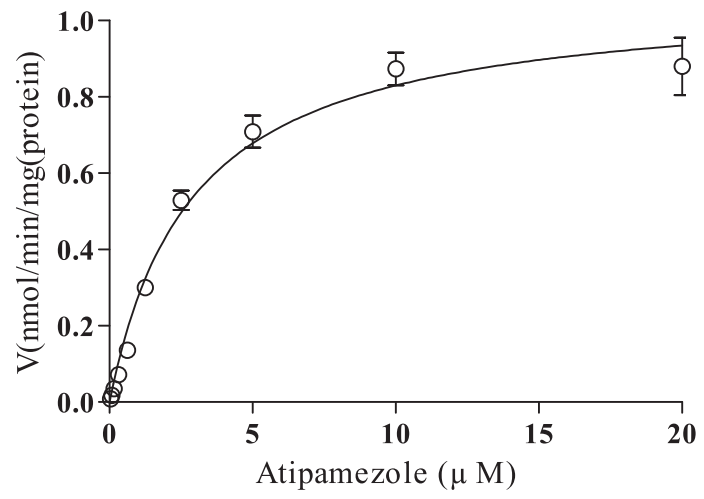

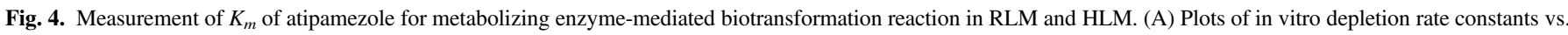

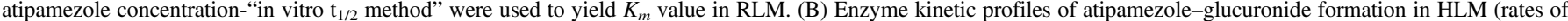

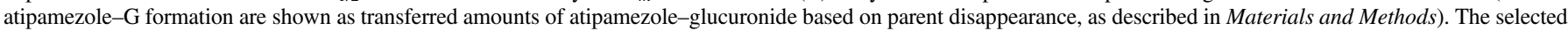
in vitro concentrations cover the in vivo plasma concentrations in rats from linear PK to nonlinear PK. The values represent as means \pm S.D. $(n=3)$.

However, no obvious changes were observed in volume of distribution (i.v. route) and dose normalized $\mathrm{C}_{\max }$ (i.m. route).

In vitro unbound $K_{m}$ value of atipamezole in RLM is $137 \mathrm{ng} / \mathrm{ml}$ $(0.65 \mu \mathrm{M})$, which is close to the unbound $\mathrm{C}_{\max }(109 \mathrm{ng} / \mathrm{ml})$ observed in rats after i.m. administration of $3 \mathrm{mg} / \mathrm{kg}$ atipamezole. The unbound $\mathrm{C}_{\max }$ was calculated from the total $\mathrm{C}_{\max }$ of $1283 \mathrm{ng} / \mathrm{ml}$ with an average $f_{\text {u,plasma }}$ of 0.085 from the present study. The nonlinear PK may be attributed from the saturation of hepatic P450 metabolism in rats. In contrast, the in vitro unbound $K_{m}$ value of atipamezole in HLM in the presence of NADPH and UDPGA was $361 \mathrm{ng} / \mathrm{ml}(1.7 \mu \mathrm{M})$, which is much higher than the unbound $\mathrm{C}_{\max }$ of $33 \mathrm{ng} / \mathrm{ml}(0.16 \mu \mathrm{M})$ reported in clinical trial at $100 \mathrm{mg}$ QD dose.

Brain Tissue Distribution. Further investigation on potential factors contributed toward the nonlinear PK behavior was focused on the drug target tissue, that is, brain to plasma ratios $\left(K_{p}\right)$ at different doses, to investigate whether there is a target-mediated drug disposition. Disproportional increase of exposure in brain was observed as the fold of concentrations normalized to the low dose increased from 1-fold to 3.9- and 17.4-fold with dose increased from 0.3 to 1 and $3 \mathrm{mg} / \mathrm{kg}$ (observed and predicted AUCs are presented in Table 6), respectively. However, the $K_{p \text {, и }}$ values stayed about 1.4-1.5 from 0.3 to $3 \mathrm{mg} / \mathrm{kg}$, suggesting a rapid equilibrium of atipamezole between brain and plasma, and the nonlinear brain concentration is mainly driven by plasma concentration.

PBPK Modeling in Rats. PBPK model was established in rats with modules of passive diffusion and metabolic clearance using the following: 1) a back-calculated $V_{\max }$ from in vivo clearance and in vitro determined $K_{m}$, and 2) distribution parameters $\left(K_{p}\right)$ determined from tissue distribution assays in various organs. Lukacova $K_{p}$ (Miller et al., 2019) method was also used to predict the $K_{p}$ values. The predicted and measured $K_{p}$ values of individual tissues (Table 2) were found to be

TABLE 4

Unbound fraction of atipamezole in rat and human plasma at the relevant concentrations in vivo

The values represent as means \pm S.D. $(n=3)$.

\begin{tabular}{lcc}
\hline Concentration $(\mu \mathrm{M})$ & Rat & Human \\
\hline 0.3 & $0.071 \pm 0.008$ & $0.068 \pm 0.016$ \\
1 & $0.095 \pm 0.005$ & $0.060 \pm 0.006$ \\
3 & $0.090 \pm 0.005$ & $0.073 \pm 0.004$ \\
\hline
\end{tabular}

statistically different, that is, measured and predicted value ratios were beyond 0.5 - to 2-fold in most of the organs. This led us to use the measured tissue-partitioning values instead of the predicted values. A comparison of PK profiles using either measured or predicted $K_{p}$ values is presented in Fig. 6, along with the observed PK from in vivo study. As one can tell, the PBPK simulation using predicted $K_{p}$ values led to poor prediction of $\mathrm{PK}$.

Figure 7 showed the simulated i.v. plasma concentration-time profiles over different doses in rats that are well matched with the observed nonlinear PK profiles. This result demonstrates that the assumptions of permeability-limited distribution and P450-mediated hepatic clearance were able to predict the nonlinearity observed in rats. This rat PBPK model was also able to predict the plasma concentration-time profiles over different doses after i.m. administration in rats. After i.v. administration, the ratios of AUC normalized to the lowest dose were 1:3.3:22 (predicted) and 1:3.8:30 (observed) for doses of 0.3, 0.9, and $3 \mathrm{mg} / \mathrm{kg}$, respectively. After i.m. administration, the corresponding ratios of AUC for predicted and observed were 1:12.8:56 and 1:12.4:75 from $0.1,1$, and $3 \mathrm{mg} / \mathrm{kg}$, respectively. Overall, the model achieved a good correlation coefficient between predicted and observed values with $r^{2}>0.86$, and the predicted plasma concentrations described by $\mathrm{AUC}_{0 \text {-inf }}$ were all within 0.89 - to 1.41 -fold of those observed values (Table 7)

PBPK Prediction in Humans and Confirmation with Clinical Observation. The PBPK model for rat was initially applied to humans with the same mechanism, that is, saturable metabolism to investigate whether nonlinear PK would be observed in humans. However, predicted human PK was linear in the clinically relevant doses $(10-100 \mathrm{mg})$. In the investigation of metabolizing enzymes involved, in vitro studies highlighted that UGTs were mainly responsible for the metabolism of atipamezole in humans, which is different from that in rats.

In addition, liver clearance can be roughly expressed by UDPGAmediated clearance. For distribution, $K_{p}$ values from rats were calibrated using human $f_{\text {u,plasma }}$ to predict $K_{p}$ in humans. This calibration was based on the observation that the predicted volume of distribution in rats is close to the actual observed value of distribution, and tissue distributions tend to have little species difference (Rowland et al., 2011). These assumptions were further confirmed by our human PBPK modeling, as the predicted plasma AUCs and volume of distribution were in good agreement with the available clinical data from three doses from the literature (Karhuvaara et al., 1990). 
A

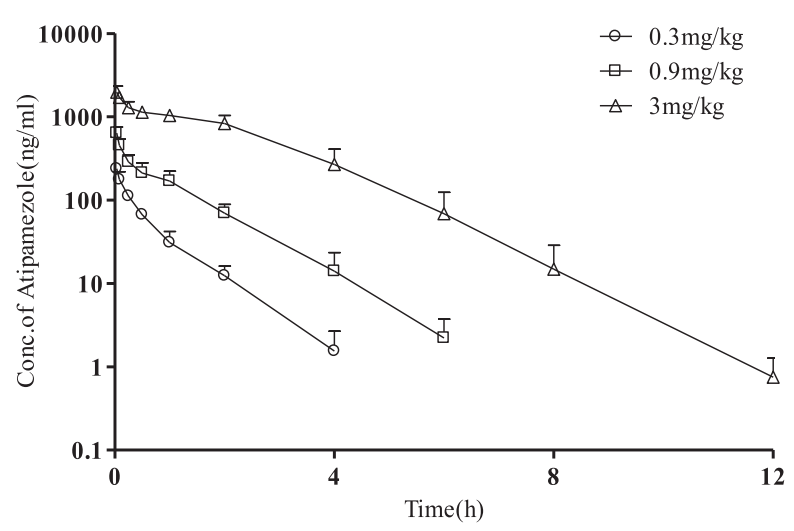

C

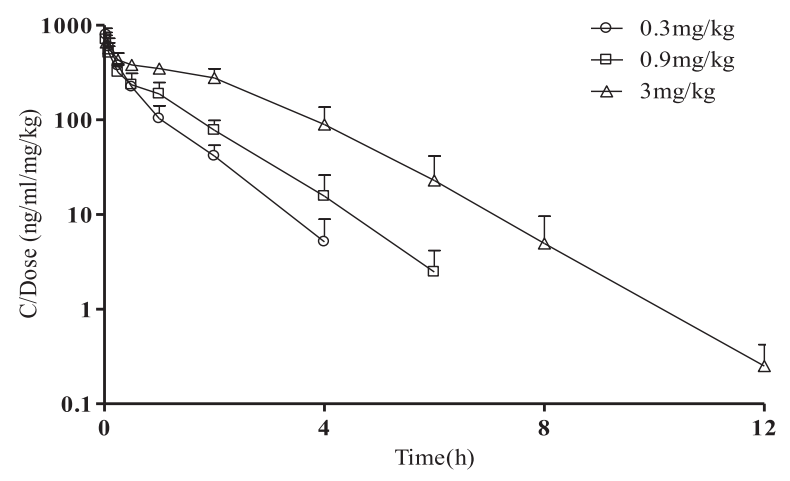

B

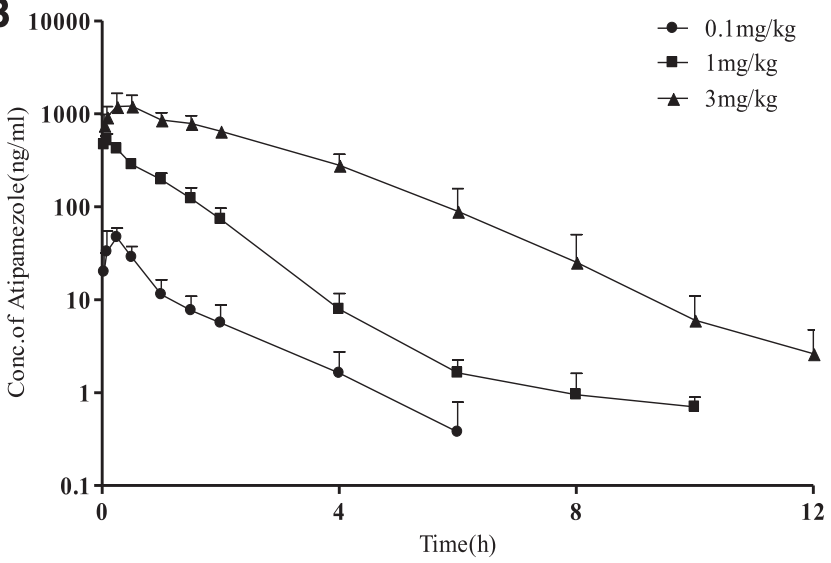

D

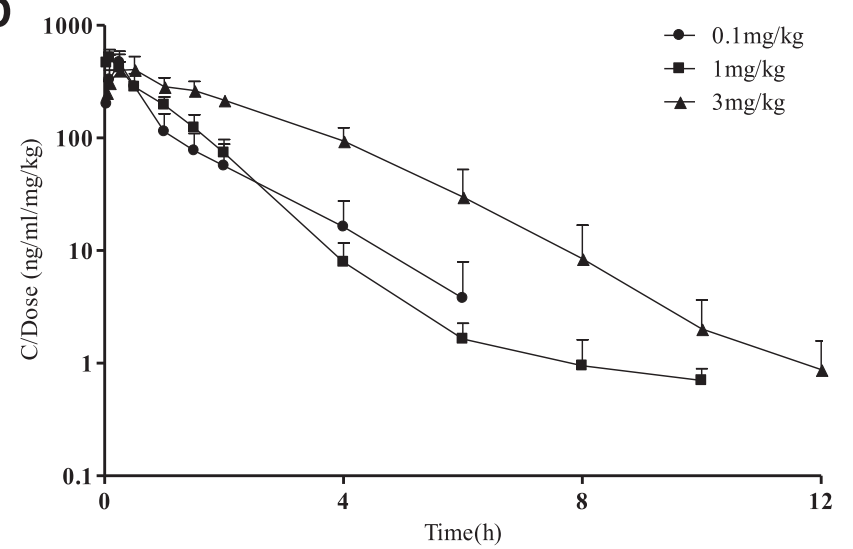

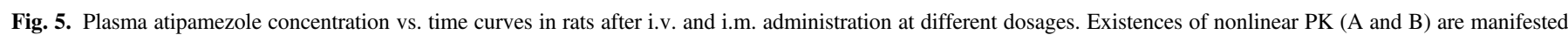
by the dose-normalized (C and D) plasma drug concentration vs. time profiles in both routes.

As mentioned above, besides using the transformed $K_{p}$ values from rats, in vitro measured $V_{\max }$ and $K_{m}$ in HLM in the present with NADPH and UDPGA and $f_{u, m i c}$ were used in our model to define the overall clearance and metabolic pathways. The observed and predicted human PK profiles for each group are presented in Fig. 8 and Table 6. These data showed the predicted PK captured the reported PK quite well with about $155 \%-175 \%$ overprediction. A few factors may contribute to this deviation, such as the sparse clinical time points and the use of in vitro hepatic clearance as the whole-body clearance. For i.m. administration, the predicted human PK was conducted at projected efficacious doses of $10-100 \mathrm{mg}$ as well as higher doses. The PK is proportional to the dose up to $1000 \mathrm{mg}$ (Fig. 9) and then becomes greater than proportional above $1000 \mathrm{mg}$.
Considering atipamezole has no species differences in its receptor affinity for $\alpha_{2}$-adrenoceptor subtypes in humans and rodents (Haapalinna et al., 1997), brain exposure in rats under pharmacological efficacious dose ( $1 \mathrm{mg} / \mathrm{kg}$, i.m.) is a practical way to bridge to human efficacious exposure. Table 7 summarized the PBPK model predicting brain exposure of atipamezole in rats and humans at different doses, along with the observed exposure from this study. Results showed AUC in human brain at $30 \mathrm{mg} /$ person is close to that in rats at $1 \mathrm{mg} / \mathrm{kg}$.

\section{Discussion}

When a drug candidate shows nonlinear PK in preclinical study, it can be a challenge to predict an appropriate efficacious dose for clinical

TABLE 5

PK parameters of atipamezole in rats after i.v. and i.m. administration determined by noncompartmental analyses The values represent as means \pm S.D. $(n=6)$.

\begin{tabular}{|c|c|c|c|c|c|c|c|}
\hline \multirow{2}{*}{ Parameter } & \multirow{2}{*}{ Unit } & \multicolumn{3}{|c|}{ i.v. } & \multicolumn{3}{|c|}{ i.m. } \\
\hline & & $0.3 \mathrm{mg} / \mathrm{kg}$ & $0.9 \mathrm{mg} / \mathrm{kg}$ & $3 \mathrm{mg} / \mathrm{kg}$ & $0.1 \mathrm{mg} / \mathrm{kg}$ & $1 \mathrm{mg} / \mathrm{kg}$ & $3 \mathrm{mg} / \mathrm{kg}$ \\
\hline$t_{1 / 2}$ & $\mathrm{~h}$ & $0.7 \pm 0.2$ & $0.8 \pm 0.1$ & $1.2 \pm 0.1$ & $1 \pm 0.1$ & $1 \pm 0.1$ & $1.2 \pm 0.1$ \\
\hline $\mathrm{AUC}_{(0-\mathrm{t})}$ & $\mathrm{h} \times \mathrm{ng} / \mathrm{ml}$ & $125.8 \pm 15.4$ & $490.4 \pm 52.6$ & $3776.3 \pm 785.7$ & $43.6 \pm 14$ & $542.8 \pm 80$ & $3281.3 \pm 636.7$ \\
\hline $\mathrm{AUC}_{(0-\infty)}$ & $\mathrm{h} \times \mathrm{ng} / \mathrm{ml}$ & $127.5 \pm 16.2$ & $493.2 \pm 51.2$ & $3780.8 \pm 779.9$ & $44.9 \pm 13.9$ & $543.8 \pm 80$ & $3284.1 \pm 638$ \\
\hline V & $\mathrm{ml} / \mathrm{kg}$ & $1624.3 \pm 162.5$ & $1931.1 \pm 257.8$ & $1497.3 \pm 161$ & & & \\
\hline $\mathrm{CL}$ & $\mathrm{ml} / \mathrm{min}$ per kilogram & $39.96 \pm 5.83$ & $30.73 \pm 3.07$ & $13.96 \pm 3.64$ & & & \\
\hline AUC/Dose & $\times 10^{-6} \mathrm{~h} \times \mathrm{kg} / \mathrm{ml}$ & $419.3 \pm 51.4$ & $544.8 \pm 58.5$ & $1258.8 \pm 261.9^{* *}$ & 449.45 & 543.79 & $1094.78 * *$ \\
\hline $\mathrm{T}_{\max }$ & $\mathrm{H}$ & & & & $0.19 \pm 0.08$ & $0.13 \pm 0.09$ & $0.46 \pm 0.27$ \\
\hline $\mathrm{C}_{\max }$ & $\mathrm{ng} / \mathrm{ml}$ & & & & $49.8 \pm 13.9$ & $553.2 \pm 75.1$ & $1283.2 \pm 354.5$ \\
\hline $\mathrm{C}_{\max } /$ Dose & $\times 10^{-6} \mathrm{~kg} / \mathrm{ml}$ & & & & 497.83 & 553.17 & 427.72 \\
\hline
\end{tabular}

$* P<0.05 ; * * P<0.01$ compared with low-dose group. 


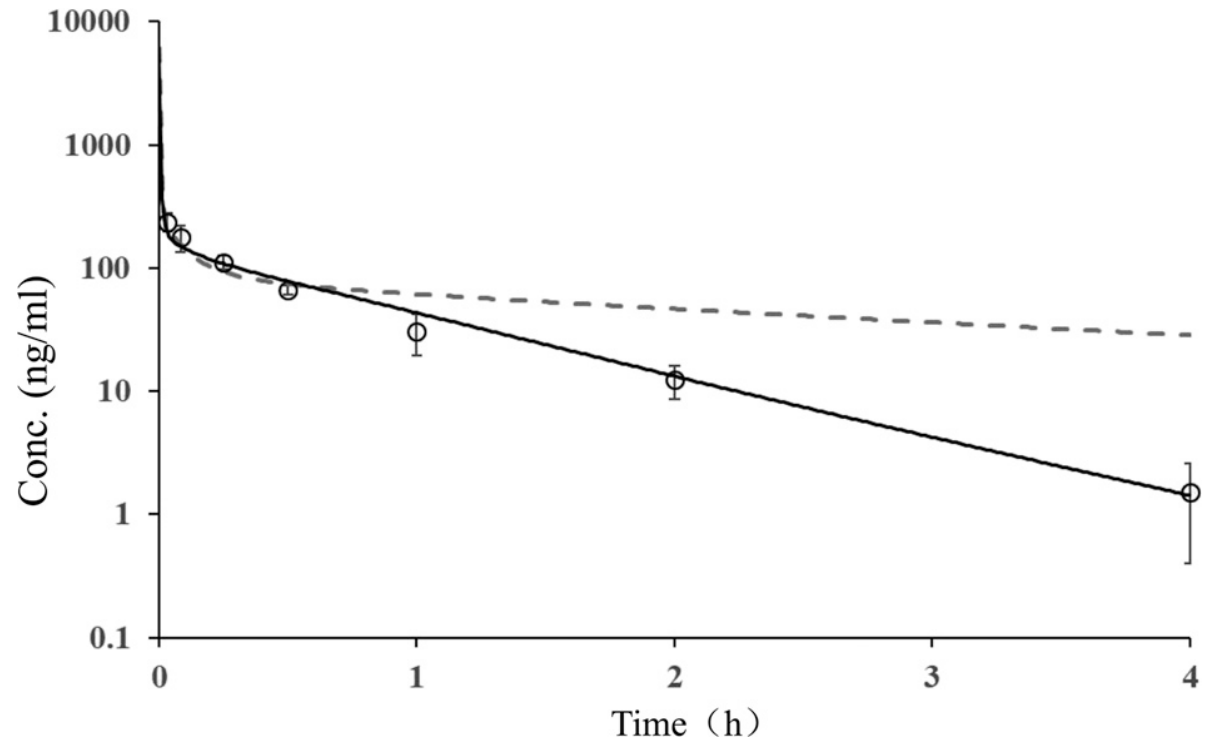

Fig. 6. Observed and predicted concentration-time profiles of atipamezole in rats after i.v. $0.3 \mathrm{mg} / \mathrm{kg}$. Black open circles represent concentrations obtained in rats; black line represents the simulated concentration-time profiles with a PBPK using measured $K_{p}$; gray dashed line represents the simulated concentration-time profiles with a PBPK using predicted $K_{p}$ generated from Lukacova method.

trials. Special attention is also required when carrying out dose escalation during phase I clinical trials. Hence, it is important to understand the factors contributing to the nonlinear PK. Nonlinear PK is believed to be mainly associated with absorption, hepatic metabolism, protein binding, liver uptake, or target tissue distribution (Ludden, 1991; Han et al., 1999; Takeuchi et al., 2001). During the preclinical PK study of atipamezole in rats, AUC normalized by the dose (AUC/dose) was found to increase markedly beyond $500 \times 10^{-6} \mathrm{~h} \times \mathrm{kg} / \mathrm{ml}$ as the dose increased. This dose-dependent nonlinear exposure is within the efficacy regiment in rats (unpublished pharmacology study data in rats). Plasma protein binding was consistent across the rats and humans at different concentrations. The drug target site of atipamezole is mainly in Central Nervous System (CNS), and brain distribution could be an additional pathway of drug disposition/elimination (Smith et al., 2018). Our results show that although brain concentration was dose-dependent superproportional increased, the $K_{p, u u}$ values were consistent across doses, suggesting that brain free concentrations were driven by the plasma free concentrations and, in turn, the efficacy. To elucidate whether liver uptake is also involved in the saturable PK of atipamezole, transport experiments with LLC-PK1/MDR1 cells were carried out. The average $\mathrm{P}_{\text {app (a-b) }}$ and $\mathrm{P}_{\text {app (b-a) }}$ of atipamezole are $80.3 \pm 5.8 \times 10^{-6}$ and $88.0 \pm$ $8.4 \times 10^{-6} \mathrm{~cm} / \mathrm{s}$ (Li et al., 2019), respectively, suggesting high permeability and passive transport of atipamezole across the biomembrane to be a major mechanism, even if an active uptake might exist. In contrast, although the $K_{p}$ values of several tissues are far higher than 1 , the $K_{p, u u}$ values were much lower than $K_{p}$ values after corrected by $f_{u}$ ( $K_{p}$,uu in Table 2$)$. Especially with low value of the $K_{\mathrm{p} \text {,uu }}$ of liver (0.06), together with high intrinsic clearance suggesting extensive metabolism occurred in liver and that attributes to low intracellular free drug concentration (Lu et al., 2006). Therefore, it suggested active uptake is unlikely to play a critical role in the mechanisms of the nonlinearity. However, it would be beneficial to further investigate this subject to
A

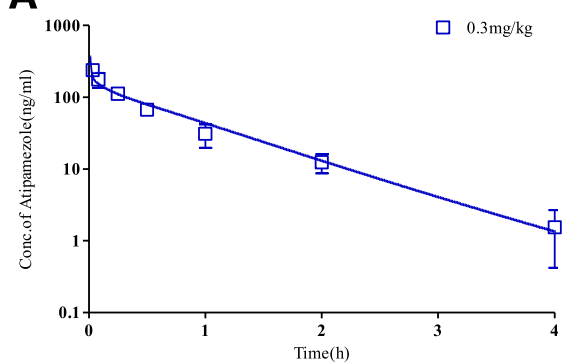

D

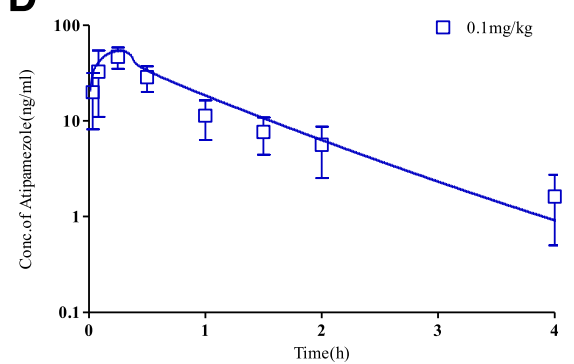

B

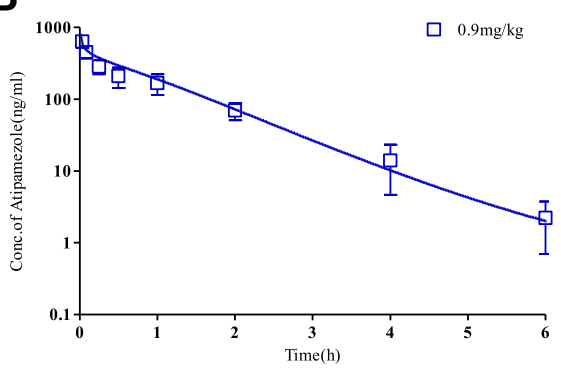

E

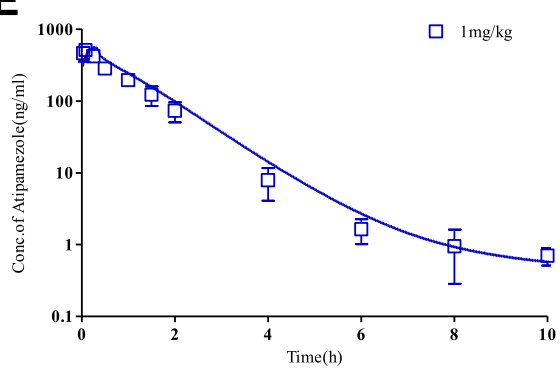

C

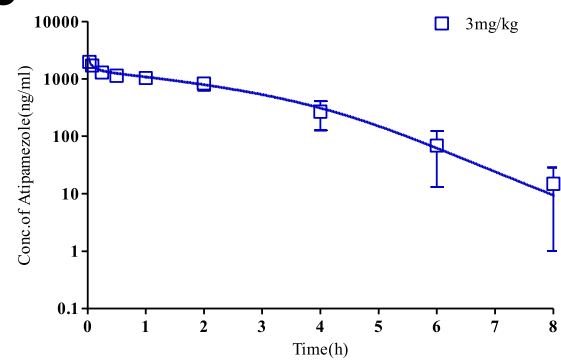

F

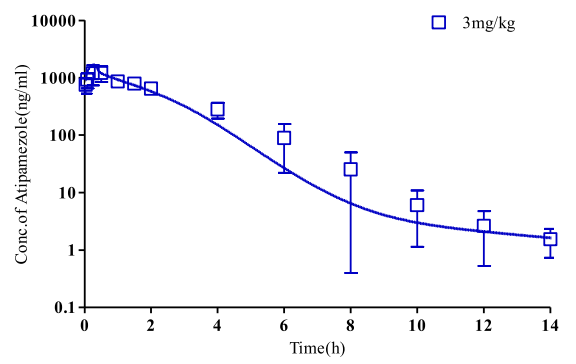

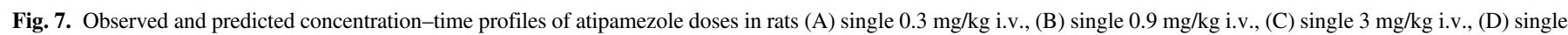

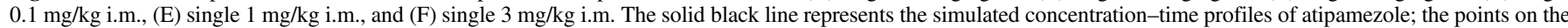
graph are the observed mean concentrations. 
TABLE 6

Predicted and observed PK parameters in rat and human receiving a single dose of atipamezole

\begin{tabular}{|c|c|c|c|c|}
\hline Species & Route and Dose & $\mathrm{AUC}_{\mathrm{ped}(0-\text {-inf })} \mathrm{h} \times \mathrm{ng} / \mathrm{ml}$ & $\mathrm{AUC}_{\mathrm{obs}(0-\text {-inf })} \mathrm{h} \times \mathrm{ng} / \mathrm{ml}$ & $\mathrm{AUC}_{\text {pre/obs }}$ Fold Error \\
\hline \multirow[t]{10}{*}{ Rat } & i.v. & & & \\
\hline & Dose $(\mathrm{mg} / \mathrm{kg})$ & & & \\
\hline & 0.3 & 148.0 & 122.2 & 1.21 \\
\hline & 0.9 & 592.8 & 479.2 & 1.24 \\
\hline & 3 & 3931.0 & 3732.1 & 1.05 \\
\hline & i.m. & & & \\
\hline & Dose $(\mathrm{mg} / \mathrm{kg})$ & & & \\
\hline & 0.1 & 52.5 & 46.1 & 1.14 \\
\hline & 1 & 641.4 & 547.3 & 1.18 \\
\hline & 3 & 2806.3 & 3289.0 & 0.85 \\
\hline \multirow[t]{5}{*}{ Human } & i.v. & & & \\
\hline & Dose (mg/person) & & & \\
\hline & 10 & 182.0 & 104.3 & 1.74 \\
\hline & 30 & 566.9 & 370.2 & 1.53 \\
\hline & 100 & 1893.5 & 1354.6 & 1.40 \\
\hline
\end{tabular}

clarify the possibility of involvement of carrier-mediated uptake, especially in humans. Mass balance study in rats showed a minimal parent recovery in feces and urine with less than $1 \%(0.28 \%)$. Metabolites of atipamezole were mainly detected in bile, suggesting the liver is the major elimination organ for atipamezole. Taken together, target site distribution has limited impact on the nonlinear PK of atipamezole, and saturation of metabolism seemed to be the main reason for nonlinear PK in rats.

In liver microsomes of rats and humans, significant difference in the hepatic clearance of atipamezole was identified. The clearance of atipamezole in rats was driven by $\mathrm{P} 450$ enzymes, whereas UGTs played a major role for the clearance in HLM. Further phenotyping study in HLM showed UGT1A4 and UGT2B10, but not other UGT isoforms, were responsible for the $\mathrm{N}$-glucuronidation of atipamezole, where UGT2B10 played a more significant role. UGT1A4 and UGT2B10 are known to specifically mediate $\mathrm{N}$-glucuronidation, especially on tertiary amine, as the N-glucuronidation on primary and secondary amines tends to not be stable. These are human liver-specific enzymes and do not present in rodents (Hawes 1998; Kaivosaari et al., 2011). Besides high abundance in humans, these enzymes are also present in dogs and monkeys with low abundance (Kaivosaari et al., 2008). UGT2B10 tends to have high affinity $\left(K_{m}\right)$ and low capability $\left(V_{\max }\right)$, whereas UGT1A4 tends to have low affinity $\left(K_{m}\right)$ and high capability $\left(V_{\max }\right)$, as reported in a study of two optical enantiomers of medetomidine: dexmedetomidine and levomedetomidine (Kaivosaari et al., 2008). We observed a similar kinetics for atipamezole with a low $K_{m}$ in HLM as
UGT2B10 played a major role. Because N-glucuronidation is a humanspecific metabolic pathway and atipamezole contains tertiary amine, this explains the observed species difference in metabolism of atipamezole between rats and humans. PBPK modeling and simulation were taken as a powerful tool to capture the dose-exposure relationship of atipamezole for both rats and humans based on reasonable assumptions, whereas species difference in metabolism made in vitro- in vivo extrapolation(IVIVE) and allometric scaling impractical. First, understanding clearance and distribution is essential for PBPK model construction. For atipamezole, identification of hepatic metabolism as the major elimination route enhanced confidence in defining and inputting clearance in our PBPK models. In addition, metabolism-related nonlinearity is often influenced by the drug plasma concentrations as well as its kinetic parameters $\left(K_{m}\right.$ and $\left.V_{\max }\right)$ for hepatic metabolism enzymes (Ludden, 1991). Determination of $K_{m}$ is usually assessed by the rate of metabolite formation against a series of substrate concentrations. However, authentic metabolites are required in this approach to quantify the formation of metabolite(s), which often is not available in drug discovery or even development stages. For atipamezole, due to multiple P450-mediated oxidation, metabolites were identified in RLM incubation and standard metabolites were not available. As such, the parent disappearance half-life assay at different atipamezole concentrations to capture the saturation of metabolism phase was applied to determine the $K_{m}$ in RLM (Obach and Reed-Hagen, 2002). A later publication provides a method to calculate the $V_{\max }$ from the same set of data (Nath and Atkins 2006). The $V_{\max }$ of atipamezole calculated from Nath and
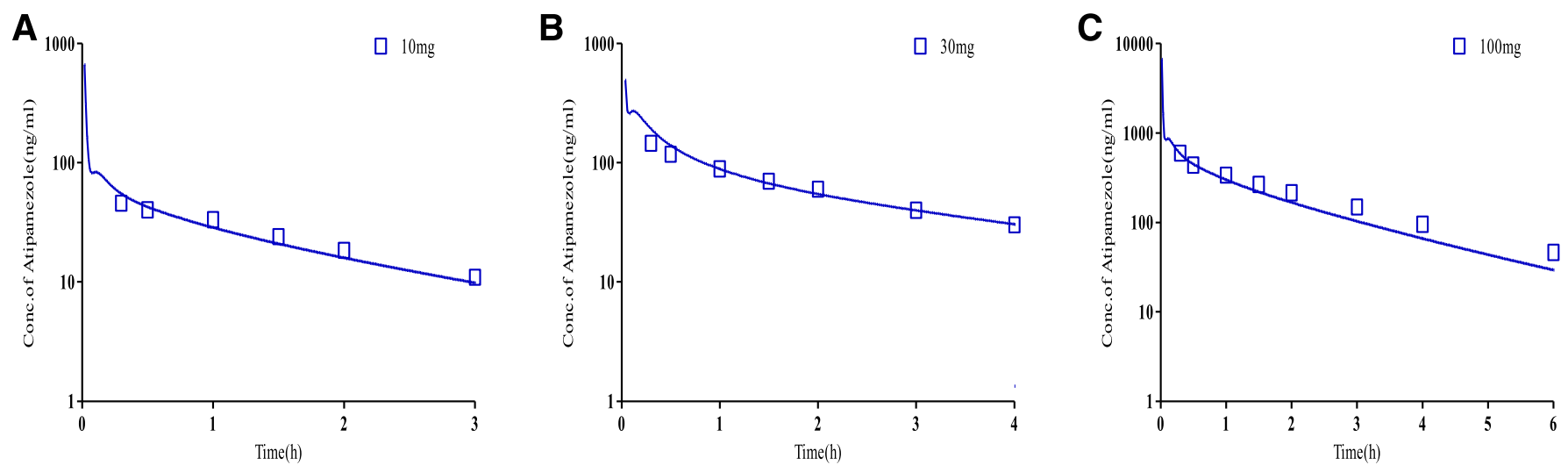

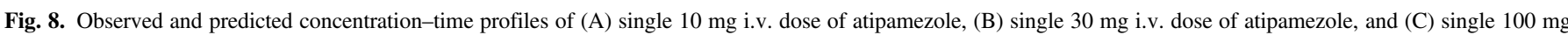

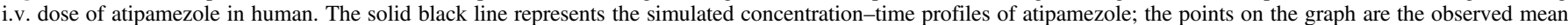
concentrations. 


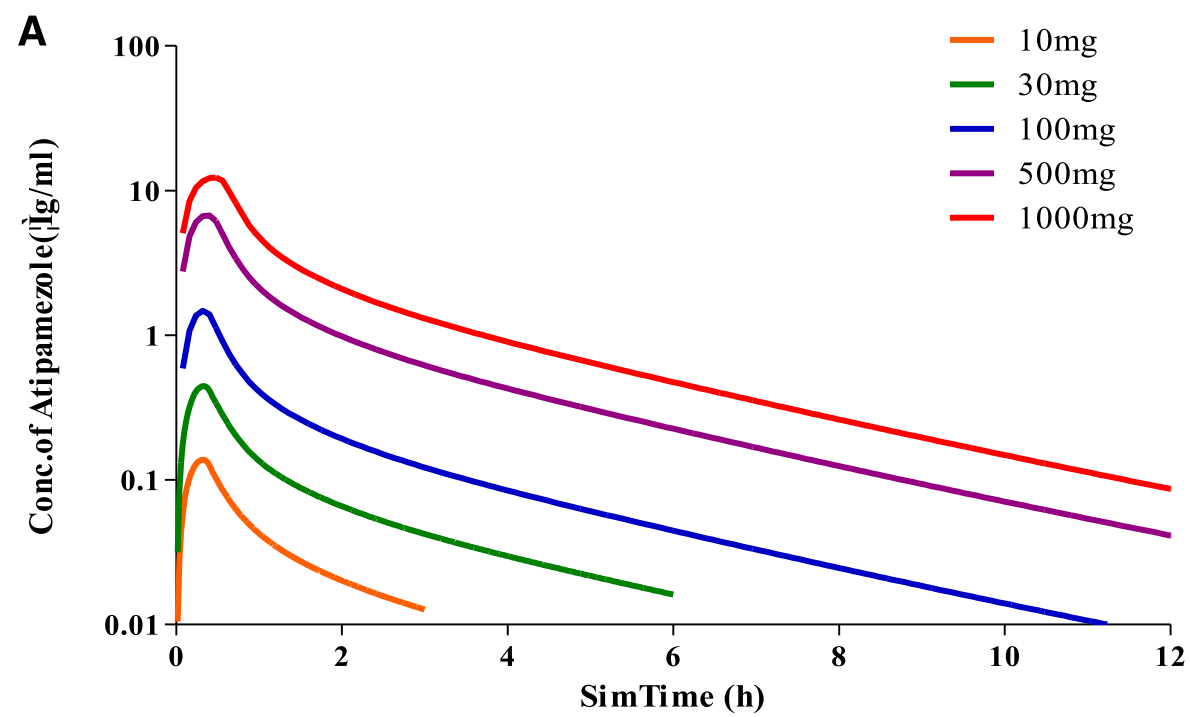

B

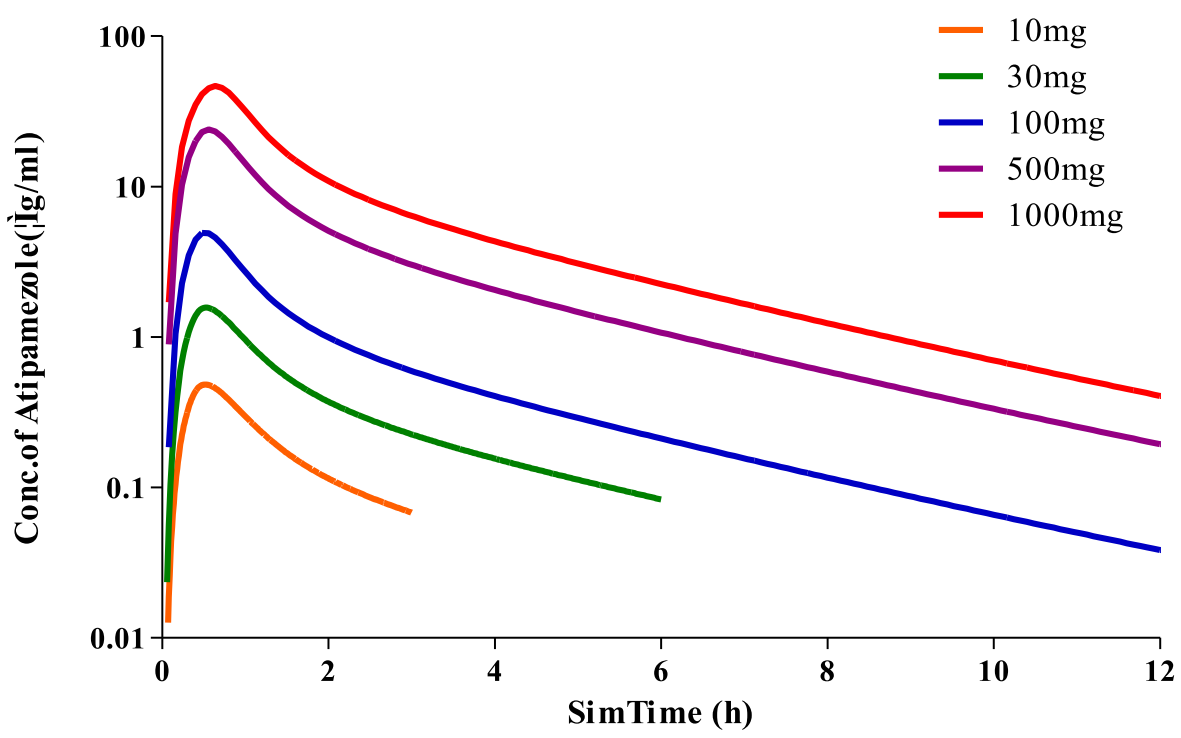

Fig. 9. Predicted dose-dependent exposure of atipamezole in Chinese. (A) Predicted concentration-time profile of atipamezole in plasma; (B) predicted concentration-time profile of atipamezole in brain.
Atkins's method was within 2-fold of that back calculated from in vivo clearance. In this study, the back-calculated $V_{\max }$ of atipamezole with a middle-out approach was used in the PBPK modeling. In HLM, because glucuronidation metabolite was found to be the dominant metabolite in a metabolite-profiling study, in vitro measured $K_{m}$ and $V_{\max }$ values toward UGT-mediated N-glucuronidation of atipamezole were directly employed in the PBPK model by a bottom-up approach.

Understanding tissue distribution is also essential for PBPK modeling. In the present study, tissue distribution experiments in rats indicated

TABLE 7

Predicted and observed brain AUC of atipamezole in rat and human receiving a single i.m. of atipamezole

\begin{tabular}{lccc}
\hline Species & Dose & $\mathrm{AUC}_{\text {ped(0-inf })} \mathrm{h} \times \mathrm{ng} / \mathrm{ml}$ & $\mathrm{AUC}_{\text {obs }(0-\text { inf })} \mathrm{h} \times \mathrm{ng} / \mathrm{ml}$ \\
\hline \multirow{4}{*}{ Rat } & $0.3 \mathrm{mg} / \mathrm{kg}$ & 650 & 703 \\
& $1 \mathrm{mg} / \mathrm{kg}$ & 2500 & 2553 \\
& $3 \mathrm{mg} / \mathrm{kg}$ & 11,500 & 16,790 \\
Human & $10 \mathrm{mg}$ & 600 & \\
& $30 \mathrm{mg}$ & 2300 & \\
& $100 \mathrm{mg}$ & 7400 & \\
\hline
\end{tabular}

atipamezole distributed rapidly to most tissues, including lung, liver, spleen, brain, adipose, kidney, heart, and muscle, and their concentration-time profiles were parallel to the blood concentrationtime curve. In the full PBPK distribution model, we therefore assumed that the drug distributes instantaneously and homogeneously within each tissue and that its uptake is limited by the blood flow (perfusion limited module in the GastroPlus). Furthermore, in the current study, transporter-mediated tissue permeation and distribution for atipamezole were not considered based on a previous study that showed atipamezole is not a substrate of P-gp ( $\mathrm{Li}$ et al., 2019). The tissue-to-blood partitioning coefficients $\left(K_{p}\right)$ were determined in rats and incorporated into the PBPK model with respective free fractions $\left(f_{\mathrm{u}, t}\right)$ for each individual tissue. Our results also show that using experimentally determined $K_{p}$ values generated better PK prediction in PBPK modeling, although software prediction based on physicochemical properties has certain merit. The $K_{p}$ values are believed to carry minimal species difference; thus, in this study, converted human $K_{p}$ values were calculated from the ratio of free concentration of atipamezole in human plasma over its free concentrations in rat tissues. The PBPK prediction using these converted $K_{p}$ values showed a good PK prediction in humans. Although i.m. administration is not a popular route in drug 
administration, it is still an effective route for first-aid administration and avoiding first-pass elimination. In the rat PBPK modeling of atipamezole via i.m. administration, selecting suspension formation in the software was found to provide a better prediction of PK profiles (e.g., $\mathrm{T}_{\max }$ and $\mathrm{C}_{\max }$ ) over the solution formation. This might be attributed to the fact that administration of a drug in solution via i.m. route would have a similar delay in absorption, as well as distribution in the body as if the drug was in suspension.

In summary, our study exemplifies that sometimes there is a cross species barrier for extrapolating animal PK to human PK. Understanding the species similarity or difference in metabolism/transport and sometimes excretion is important in the preclinical space for the prediction of PK or DDI risks in human. This study demonstrates a PBPK model that can capture nonlinear PK in rat with both i.v. and i.m. dosing routes. It provides insights into potential clinical application of atipamezole via i.v. or i.m. administration routes.

\section{Acknowledgments}

We greatly appreciate the help of Dr. Jennifer Fretland in editing this manuscript.

\section{Authorship Contributions}

Participated in research design: $\mathrm{Li}, \mathrm{Su}, \mathrm{Lu}$, Zhuang.

Conducted experiments: Li, Gao, Yang, Xiang, T Zhang.

Contributed new reagents or analytic tools: Su.

Performed data analysis: Zhuang, Li, W Zhang.

Wrote or contributed to the writing of the manuscript: Zhuang, $\mathrm{Li}, \mathrm{Lu}$.

\section{References}

Bachmann KA and Belloto RJ Jr (1999) Differential kinetics of phenytoin in elderly patients. Drugs Aging 15:235-250.

Chen T, Mager DE, and Kagan L (2013) Interspecies modeling and prediction of human exenatide pharmacokinetics. Pharm Res 30:751-760.

Chirehwa MT, Rustomjee R, Mthiyane T, Onyebujoh P, Smith P, McIlleron H, and Denti P (2015) Model-based evaluation of higher doses of rifampin using a semimechanistic model in corporating autoinduction and saturation of hepatic extraction. Antimicrob Agents Chemother $\mathbf{6 0}$ : 487-494.

Davies B and Morris T (1993) Physiological parameters in laboratory animals and humans. Pharm Res 10:1093-1095.

Dong JQ, Salinger DH, Endres CJ, Gibbs JP, Hsu CP, Stouch BJ, Hurh E, and Gibbs MA (2011) Quantitative prediction of human pharmacokinetics for monoclonal antibodies: retrospective analysis of monkey as a single species for first-in-human prediction. Clin Pharmacokinet 50:131-142.

Ewing KK, Mohammed HO, Scarlett JM, and Short CE (1993) Reduction of isoflurane anesthetic requirement by medetomidine and its restoration by atipamezole in dogs. Am J Vet Res 54:294-299.

Fukuchi Y, Toshimoto K, Mori T, Kakimoto K, Tobe Y, Sawada T, Asaumi R, Iwata T, Hashimoto Y, Nunoya KI, et al. (2017) Analysis of nonlinear pharmacokinetics of a highly albumin-bound compound: contribution of albumin-mediated hepatic uptake mechanism. J Pharm Sci 106:2704-2714.

Haapalinna A, Viitamaa T, MacDonald E, Savola JM, Tuomisto L, Virtanen R, and Heinonen E (1997) Evaluation of the effects of a specific alpha 2-adrenoceptor antagonist, atipamezole, on alpha 1- and alpha 2-adrenoceptor subtype binding, brain neurochemistry and behaviour in comparison with yohimbine. Naunyn Schmiedebergs Arch Pharmacol 356:570-582.

Han YH, Kato Y, and Sugiyama Y (1999) Nonlinear disposition kinetics of a novel antifolate, MX68, in rats. J Pharmacol Exp Ther 291:204-212.

Hawes EM (1998) N+-glucuronidation, a common pathway in human metabolism of drugs with a tertiary amine group. Drug Metab Dispos 26:830-837.

Houston JB (1994) Utility of in vitro drug metabolism data in predicting in vivo metabolic clearance. Biochem Pharmacol 47:1469-1479.

Huang SM and Rowland M (2012) The role of physiologically based pharmacokinetic modeling in regulatory review. Clin Pharmacol Ther 91:542-549.

Jamei M, Dickinson GL, and Rostami-Hodjegan A (2009) A framework for assessing interindividual variability in pharmacokinetics using virtual human populations and integrating general knowledge of physical chemistry, biology, anatomy, physiology and genetics: a tale of 'bottom-up' vs 'top-down' recognition of covariates. Drug Metab Pharmacokinet 24:53-75.

Jones HM, Chen Y, Gibson C, Heimbach T, Parrott N, Peters SA, Snoeys J, Upreti VV, Zheng M, and Hall SD (2015) Physiologically based pharmacokinetic modeling in drug discovery and development: a pharmaceutical industry perspective. Clin Pharmacol Ther 97:247-262.
Kaivosaari S, Finel M, and Koskinen M (2011) N-glucuronidation of drugs and other xenobiotics by human and animal UDP-glucuronosyltransferases. Xenobiotica 41:652-669.

Kaivosaari S, Toivonen P, Aitio O, Sipilä J, Koskinen M, Salonen JS, and Finel M (2008) Regioand stereospecific N-glucuronidation of medetomidine: the differences between UDP glucuronosyltransferase (UGT) 1A4 and UGT2B10 account for the complex kinetics of human liver microsomes. Drug Metab Dispos 36:1529-1537.

Karhuvaara S, Kallio A, Scheinin M, Anttila M, Salonen JS, and Scheinin H (1990) Pharmacological effects and pharmacokinetics of atipamezole, a novel alpha 2-adrenoceptor antagonist-a randomized, double-blind cross-over study in healthy male volunteers. Br J Clin Pharmaco 30:97-106.

Li M, Zhao P, Pan Y, and Wagner C (2018a) Predictive performance of physiologically based pharmacokinetic models for the effect of food on oral drug absorption: current status. $C P T$ Pharmacometrics Syst Pharmacol 7:82-89.

Li Z, Gao Y, Yang C, Qiu X, Zhang T, Zhang W, Su R, and Zhuang X (2018b) In vitro metabolism of atipamezole in different species of liver microsomes and cytochrome P450 enzymes by highresolution mass spectrometry. Zhongguo Yaolixue Yи Dulixue Zazhi 32:946-952.

Li Z, Zhang Y, Gao Y, Xiang Y, Zhang W, Lu C, and Zhuang X (2019) Atipamezole is a promising non-discriminative inhibitor against pan-CYP450 including diclofenac $4^{\prime}$-hydroxylation: a comparison with ABT for drug ADME optimization and mechanism study. Eur J Pharm Sci 130:156-165.

Liu F, Zhuang X, Yang C, Li Z, Xiong S, Zhang Z, Li J, Lu C, and Zhang Z (2014) Characterization of preclinical in vitro and in vivo ADME properties and prediction of human PK using a physiologically based pharmacokinetic model for YQA-14, a new dopamine D3 receptor antagonist candidate for treatment of drug addiction. Biopharm Drug Dispos 35:296-307.

Lu C, Li P, Gallegos R, Uttamsingh V, Xia CQ, Miwa GT, Balani SK, and Gan LS (2006) Comparison of intrinsic clearance in liver microsomes and hepatocytes from rats and humans: evaluation of free fraction and uptake in hepatocytes. Drug Metab Dispos 34:1600-1605.

Ludden TM (1991) Nonlinear pharmacokinetics: clinical implications. Clin Pharmacokinet 20: 429-446.

Miller NA, Reddy MB, Heikkinen AT, Lukacova V, and Parrott N (2019) Physiologically based pharmacokinetic modelling for first-in-human predictions: an updated model building strategy illustrated with challenging industry case studies. Clin Pharmacokinet 58:727-746.

Nath A and Atkins WM (2006) A theoretical validation of the substrate depletion approach to determining kinetic parameters. Drug Metab Dispos 34:1433-1435.

Obach RS and Reed-Hagen AE (2002) Measurement of Michaelis constants for cytochrome P450mediated biotransformation reactions using a substrate depletion approach. Drug Metab Dispos 30:831-837.

Pertovaara A, Haapalinna A, Sirviö J, and Virtanen R (2005) Pharmacological properties, central nervous system effects, and potential therapeutic applications of atipamezole, a selective alpha2 adrenoceptor antagonist. CNS Drug Rev 11:273-288.

Pertovaara A, Linnankoski I, Artchakov D, Rämä P, and Carlson S (2004) A potential aphrodisiac for female macaques. Pharmacol Biochem Behav 79:137-141.

Pitkänen A, Narkilahti S, Bezvenyuk Z, Haapalinna A, and Nissinen J (2004) Atipamezole, an alpha(2)-adrenoceptor antagonist, has disease modifying effects on epileptogenesis in rats. Epilepsy Res 61:119-140.

Puurunen K, Jolkkonen J, Sirviö J, Haapalinna A, and Sivenius J (2001) An $\alpha(2)$-adrenergic antagonist, atipamezole, facilitates behavioral recovery after focal cerebral ischemia in rats. Neuropharmacology 40:597-606.

Rowland M, Peck C, and Tucker G (2011) Physiologically-based pharmacokinetics in drug development and regulatory science. Anпи Rev Pharmacol Toxicol 51:45-73.

Shebley M, Sandhu P, Emami Riedmaier A, Jamei M, Narayanan R, Patel A, Peters SA, Reddy VP, Zheng M, de Zwart L, et al. (2018) Physiologically based pharmacokinetic model qualification and reporting procedures for regulatory submissions: a consortium perspective. Clin Pharmacol Ther 104:88-110.

Smith DA, van Waterschoot RAB, Parrott NJ, Olivares-Morales A, Lavé T, and Rowland M (2018) Importance of target-mediated drug disposition for small molecules. Drug Discov Today 23 2023-2030.

Tachibana T, Kato M, and Sugiyama Y (2012) Prediction of nonlinear intestinal absorption of CYP3A4 and P-glycoprotein substrates from their in vitro $\mathrm{K}_{\mathrm{m}}$ values. Pharm Res 29:651-668.

Takeuchi T, Tagawa Y, Hagihara K, Maeshiba Y, Yamashita K, Tsukuda R, and Yoshimura Y (2001) Nonlinear pharmacokinetics of TAK-044, a new endothelin antagonist, in rats. Biopharm Drug Dispos 22:221-230.

Tang H and Mayersohn M (2006) A global examination of allometric scaling for predicting human drug clearance and the prediction of large vertical allometry. J Pharm Sci 95:1783-1799.

Viitamaa T, Haapalinna A, and Heinonen E (1995) The effect of the á2-adrenoceptor antagonist, atipamezole, on the sexual behavior of sexually low-active male rats. Behav Pharmacol 6: 634-635.

Wu F, Gaohua L, Zhao P, Jamei M, Huang SM, Bashaw ED, and Lee SC (2014) Predicting nonlinear pharmacokinetics of omeprazole enantiomers and racemic drug using physiologically based pharmacokinetic modeling and simulation: application to predict drug/genetic interactions. Pharm Res 31:1919-1929.

Zhuang X and Lu C (2016) PBPK modeling and simulation in drug research and development. Acta Pharm Sin B 6:430-440.

Address correspondence to: Dr. Xiaomei Zhuang, State Key Laboratory of Toxicology and Medical Countermeasures, Beijing Institute of Pharmacology and Toxicology, Beijing 100850, China. E-mail: xiaomeizhuang@163.com 